\title{
Effects of Radix Total Glucosides of Paeonia on the Endoplasmic Reticulum Stress and Macrophage Recruitment on Diabetic Cardiomyopathy Rats
}

\author{
Hongyu Kang ${ }^{1, a}$ Ximin Liu ${ }^{2, b}$ Yaping Zheng ${ }^{3, ~ c}$ Chunjie Liu ${ }^{1, d}$ Jingli Ren ${ }^{1, e}$ \\ ${ }^{1}$ Department of Pharmacology, luohe medical college, Luohe, Henan 462002, China \\ ${ }^{2}$ Department of anatomy, luohe medical college, Luohe, Henan 462002, China \\ ${ }^{3}$ Department of physiology, luohe medical college, Luohe, Henan 462002, China \\ ${ }^{a}$ email, ${ }^{b}$ email, ${ }^{c}$ email, ${ }^{\text {eemail, }}{ }^{\mathrm{e} e m a i l,}$
}

\begin{abstract}
Keywords: Ischemia Reperfusion; Total Glucosides of Paeonia; Apoptosis; Wnt3a; P-GSK-3B; B-Catenin
\end{abstract}

\begin{abstract}
Aim To investigate the effects of Total glucosides of paeonia (TGP) on on myocardial ischemia reperfusion rats and the role of $\mathrm{Wnt} / \beta$-catenin signaling pathway was also investigated. Methods Sprague-Dawley rats were randomly divided into 5 groups: sham operation group, IR group, TGP low-dose group(i.g.50mg/kg), TGP middle-dose group(i.g.100mg/kg), TGP high-dose

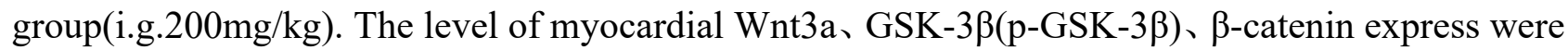
measureed by western blot. Myocardial infarction size was detected by TTC staining. The apoptotic cardiomyocytes were assessed through TUNEL .Results Compared with IR group, TGP can increase LVSP, LVEDP, +dp/dtmax, LVEF. The expressions of Wnt3a,GSK-3 $\beta$ (p-GSK-3 $\beta$ ), $\beta$-catenin, the apoptotic index and myocardial infarction size were lower in the TGP group than those in I/R group $(\mathrm{P}<0.01)$. Conclusion The protective effect of TGP on myocardial ischemia reperfusion rats is obviously, Wnt/ $\beta$-catenin signaling pathway maybe involved in the mechanism.
\end{abstract}

\section{Introduction}

Reperfusion after myocardial ischemia is the most effective way to reduce myocardial infarct size, but myocardial ischemia-reperfusion (I / R) injury can also lead to serious myocardial injury. Cardiovascular surgery is an important reason for complications, manifested as decreased cardiac function, arrhythmia and myocardial infarction and so on.

The total glucosides of paeony (TGP) is an effective component of traditional Chinese medicine extract from Paeoniae Alba. It has anti-inflammatory, immunoregulatory and anti-oxidative effects, and TGP has obvious protective effect on heart, brain and liver ischemia $[1,2,3]$, and this effect may be related to its antioxidant, but few domestic and foreign literature Wnt / $\beta$-catenin signaling pathway in anti-ischemia-reperfusion injury in the role. In this study, Wnt / $\beta$-catenin signal pathway to study the effect of total glucosides of paeony on myocardial ischemia-reperfusion injury in myocardial infarction and apoptosis.

\section{Materials and Methods}

Experimental Animal. Body weight $200 \sim 250$ g clean grade female SD rats, by the Zhengzhou University Medical Center Experimental Animal Center to provide license number: SCXK (Yu) 2010-0002.

Reagent. The total glucosides of paeony (Sanjiu Pharmaceutical Co., Ltd. Lot number H19980147); TUNEL apoptosis detection kit (Roche company batch number 2101401); rabbit anti-wnt3a antibody (Beijing Bo Aosen Biotechnology Co., Ltd.); rabbit anti-B-catenin (Beijing Pharmaceutical Co., Ltd.); Rabbit anti-p-GSK-3p (Ser9) antibody (Cell Signaling Co., Ltd.).

Myocardial Ischemia-Reperfusion Model Preparation and Animal Group. The male SD rats, anesthesia, supine fixed to monitor the heart of the ECG machine. The middle of the anterior central 
incision, endotracheal intubation, then artificial respiration machine, cut 3, 4 ribs, pull the chest wall with a pull, in both sides of the chest wall muscles were wearing a pair of lines to stay in the inner circle. The left anterior wall of the left atrial appendage $3 \sim 4 \mathrm{~mm}$, with 5-0 non-invasive suture needle oblique to the upper right pulmonary artery cone direction needle, and set a groove latex Tube spare, respectively, the line ends into a small circle. After ligating the left coronary artery, the left anterior descending coronary artery was ligated for $30 \mathrm{~min}$, and then ligated for $30 \mathrm{~min}$. Then, the ligating line was ligated for $120 \mathrm{~min}$, and then the myocardium was ligated with the latex tube. In the sham - operated group, only the left anterior descending coronary artery was isolated without ligating the left anterior descending coronary artery.

Rats were randomly divided into sham operation group, Ischemia reperfusion group (IR) group and low dose paeoniflorin group $(\mathrm{P}<0.05)$. The rats were pretreated with paeoniflorin $(20 \mathrm{mg} / \mathrm{ml}$ in saline) The rats of sham operation group and ischemia-reperfusion group were given normal saline $(1 \mathrm{ml} / \mathrm{d})$ once a day for 1 time. All animals were sacrificed at the end of the administration. All hearts were sacrificed and the hearts were quickly frozen in liquid nitrogen.

Cardiac Function Determination. Preparation of cardiac function model, at the same time, the right carotid artery cannulation to the left ventricle in rats, the pressure sensor connected to BL420 biological signal acquisition and analysis system to record left ventricular systolic pressure (left ventricular systolic pressure, left ventricular end-diastolic Left ventricular end diastolic pressure (LVEDP), left ventricular systolic pressure $(+\mathrm{dp} / \mathrm{dtmax})$ and maximal diastolic pressure $(-\mathrm{dp} /$ dtmax).

Determination of Myocardial Infarct Size. The frozen heart was placed in $-20{ }^{\circ} \mathrm{C}$ frozen $1 \mathrm{~h}$, cut into $2 \mathrm{~mm}$ thick slices, placed in $1 \%$ chlorinated triphenyl tetrazolium chloride (TTC) phosphate buffer, $37{ }^{\circ} \mathrm{C}$ avoid After incubation for $20 \mathrm{~min}$, the sections were fixed in formaldehyde solution at a concentration of $10 \%$ overnight. The non-myocardial infarction area was stained dark red due to reduced TTC. The infarct area was grayish white. The area of left ventricle and infarct area was measured by image analysis system, and the percentage of infarct area was calculated as infarct area of left ventricular tissue slice and total area of left ventricular tissue slice.

TUNEL Method to Detect Myocardial Apoptosis. Apoptosis myocardial tissue paraffin sections, according to kit instructions, the use of deoxyribonucleotides transferase-mediated nick end labeling (TUNEL method) detection of myocardial cell apoptosis. The nuclei of apoptotic cells were stained brown under light microscope. The number of apoptotic cells was calculated as the percentage of the number of apoptotic cells by counting at least 5 high power fields and not less than 200 myocardium nuclei.

Western blot analysis the expression of wnt3a, p-GSK-3 $\beta$ and $\beta$-catenin in cardiac tissue was measured by tissue culture. The tissue samples were harvested and centrifuged for $30 \mathrm{~min}$. SDS-PAGE electrophoresis, $1 \mu \mathrm{g}$ protein denaturation after cooling, add sample, electrophoresis, transfer membrane; transfer membrane plus blocking solution, closed at room temperature 120min; by adding rabbit anti-wnt3a antibody (1: 500), rabbit anti- (1: 1000) and rabbit anti- $\beta$-catenin antibody (1: 1000 dilution), and incubated overnight at $4^{\circ} \mathrm{C}$ in a shaker. After washing, alkaline phosphatase-conjugated goat anti-rabbit IgG antibody Diluted) $37{ }^{\circ} \mathrm{C}$ incubation for one hour; rinse with coloring agent, dark color $2 \sim 5 \mathrm{~min}$, the band appears to terminate the reaction.

Statistical Analysis. Using SPSS10.0 statistical software, measurement data using the s description, the comparison between groups using analysis of variance, $\mathrm{P}<0.05$ indicates a statistically significant.

\section{Results}

The Cardiac Function of Rats in Each Group. LVEDP, LVSP, $\pm \mathrm{dp} / \mathrm{dtmax}$ and LVEF in IR group were significantly higher than those in sham-operated group $(\mathrm{P}<0.01)$. The effect of total glucosides of paeony on concentration-dependently decreased the cardiac function of IR rats, IR group $(\mathrm{P}<0.05)$ (Table 1). 
Table.1 LVSP、LVEDP、 \pm dp/dtmax LVEF in different groups $\left(\bar{x} \pm_{\mathrm{s}}\right)$

\begin{tabular}{cccccc}
\hline Group & $\mathrm{n}$ & $\begin{array}{c}\text { LVSP } \\
(\mathrm{mmHg})\end{array}$ & $\begin{array}{c}\text { LVEDP } \\
(\mathrm{mmHg})\end{array}$ & $\begin{array}{c}+\mathrm{dp} / \mathrm{dtmax} \\
(\mathrm{mmHg} / \mathrm{s})\end{array}$ & $\begin{array}{c}-\mathrm{dp} / \mathrm{dtmax} \\
(\mathrm{mmHg} / \mathrm{s})\end{array}$ \\
\hline $\begin{array}{c}\text { Sham operation } \\
\text { group }\end{array}$ & 12 & $148.32 \pm 9.08$ & $7.86 \pm 0.51$ & $4885.41 \pm 38.35$ & $4440.25 \pm 42.48$ \\
IR group & 15 & $78.66 \pm 6.14^{* *}$ & $12.88 \pm 0.35^{* *}$ & $3670.24 \pm 35.17^{* *}$ & $3158.34 \pm 42.31^{* *}$ \\
$\begin{array}{c}\text { Low dose } \\
\text { Moderate dose }\end{array}$ & 15 & $88.23 \pm 5.02^{* *}$ & $10.68 \pm 0.48^{* * \#}$ & $3911.74 \pm 32.66^{* \#}$ & $3526.21 \pm 40.397^{* * \#}$ \\
High dose & 13 & $113.25 \pm 3.15^{* * \#}$ & $9.48 \pm 0.23^{* * \#}$ & $4234.26 \pm 35.14^{* \# \#}$ & $3921.58 \pm 36.36^{* * \#}$ \\
\hline
\end{tabular}

The Wnt3a, Phosphorylated GSK-3B (P-GSK-3B) and B-Catenin Protein Expression in Each Group. Compared with the normal control group, the expression of wnt3a, p-GSK-3 $\beta$ and $\beta$-catenin was significantly increased in IR group (all $\mathrm{P}<0.01$ ), while the total glucosides of paeony in concentration-dependently inhibited wnt3a, $\mathrm{p}$-GSK B $\beta$ and $\beta$-catenin protein expression $(\mathrm{P}<0.05)$

Table 2 The expression of wnt3a, p-GSK-3 $\beta$ and $\beta$-catenin protein in each group

\begin{tabular}{ccccc}
\hline Group & $\mathrm{n}$ & wnt3a & p-GSK-3 $\beta$ & $\beta$-catenin \\
\hline Normal control group & 20 & $0.31 \pm 0.08$ & $0.48 \pm 0.03$ & $0.28 \pm 0.06$ \\
IR group & 18 & $1.32 \pm 0.19^{* *}$ & $1.24 \pm 0.15^{* *}$ & $0.44 \pm 0.08^{* *}$ \\
Low dose & 18 & $1.08 \pm 0.15^{* * \#}$ & $0.84 \pm 0.16^{* * \#}$ & $0.40 \pm 0.08^{* *}$ \\
Moderate dose & 18 & $0.83 \pm 0.16^{* * \#}$ & $0.73 \pm 0.18^{* * \#}$ & $0.35 \pm 0.07^{* \#}$ \\
High dose & 18 & $0.55 \pm 0.13^{* \# \#}$ & $0.58 \pm 0.17^{* \# \#}$ & $0.33 \pm 0.05^{* \#}$
\end{tabular}

Compared with the sham-operation group, $* \mathrm{P}<0.05, * * \mathrm{P}<0.01$ compared with the IR group, $* \mathrm{P}$ $<0.05, * * \mathrm{P}<0.01$

Myocardial Apoptosis and Myocardial Infarction Area. Normal myocardium nucleus blue; apoptotic nuclei of different shades of brown. Compared with sham-operated group, the apoptotic rate of IR group was significantly higher than that of sham-operated group $(\mathrm{P}<0.01)$; the apoptosis rate of low, medium and high dose paeoniflorin pretreatment decreased in a dose-dependent manner $(\mathrm{P}<0.05)$. In the sham operation group, no myocardial infarct was found, and the percentage of infarcted area in the other groups was significantly higher than that in the sham operation group (P $<0.05)$. Compared with the IR group, the percentage of infarcted area of low, medium and high dose of total glucosides of paeony preconditioning decreased. 

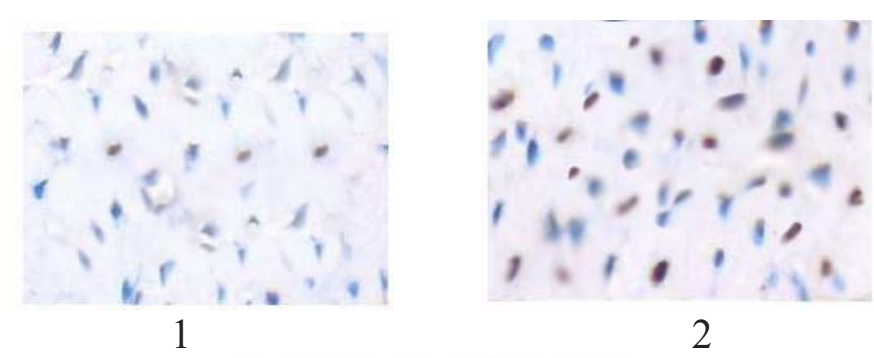

2

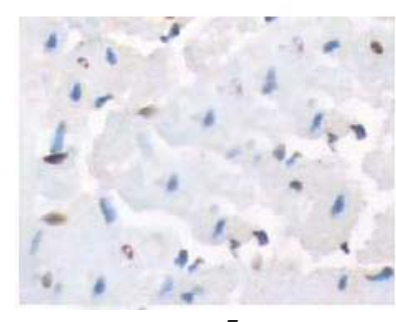

5

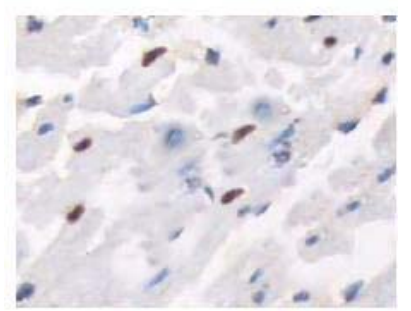

4

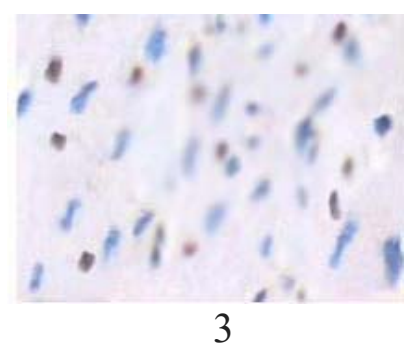

Fig 1 Apoptosis of cardiomyocytes in each group by TUNEL method $(\times 400)$

1. sham operation group; 2. IR group; 3. low dose group of total glucosides of paeony; 4. middle dose group of total glucosides of paeony; 5 . high dose group of total glucosides of paeony

Table 3 The expression in five groups

\begin{tabular}{cccc}
\hline Group & $\mathrm{n}$ & $\begin{array}{c}\text { Myocardial apoptosis } \\
(\%)\end{array}$ & Infarct size (\%) \\
\hline Sham operation group & 20 & $2.08 \pm 0.37$ & $0.00 \pm 0.00$ \\
IR group & 18 & $25.17 \pm 3.11^{* *}$ & $38.32 \pm 8.32^{* *}$ \\
Low dose & 18 & $18.24 \pm 2.58^{* * \#}$ & $31.47 \pm 7.41^{* * \#}$ \\
Moderate dose & 18 & $10.48 \pm 3.71^{* * \#}$ & $23.56 \pm 5.75^{* * \#}$ \\
High dose & 18 & $6.47 \pm 1.085^{* \# \#}$ & $14.13 \pm 4.35^{* * \#}$ \\
\hline
\end{tabular}

Compared with the sham-operation group, ${ }^{*} \mathrm{P}<0.05, * * \mathrm{P}<0.01$ compared with the IR group, * $\mathrm{P}<0.05, * * \mathrm{P}<0.01$

\section{Discussion}

Ischemic preconditioning refers to repeated, transient ischemia in the subsequent myocardial ischemia in the follow-up to be protected, so that the scope of myocardial infarction reduced, some drug pretreatment can also play a similar ischemic preconditioning myocardial protection Effect, is one of the direction of myocardial protection research. The main component of paeoniflorin, through its antioxidant effect against myocardial ischemia and hypoxia, improve cardiac function [2], the results also confirmed that the total glucosides of paeony (paeoniflorin) and the decrease of $+\mathrm{dP} / \mathrm{dtmax}$ and-dP / dtmax in myocardial ischemia-reperfusion model, which indicated the protective effect on myocardial ischemia-reperfusion.

Wnt / $\beta$-catenin signal transduction pathway is involved in the regulation of cell proliferation, migration, differentiation and apoptosis. Wnt3a is the initiation factor of Wnt / $\beta$-catenin pathway. Wnt3a protein phosphorylates $\beta$-catenin by inhibiting the activity of GSK-3 $\beta$, so that $\beta$-catenin is stable and aggregated in the cytoplasm, and finally induces the expression of target genes, thus play the corresponding biological effects [4].

Wnt signaling pathway signaling molecules can regulate the differentiation of human induced pluripotent stem cells (hPSCs) into cardiomyocytes [5]. Lian et al [6] found that by controlling the expression of GSK-3 $\beta$ and $\beta$-catenin, Or through the compound transient inhibition of Wnt signaling pathway, can efficiently induce a variety of hiPS produce functional cardiomyocytes, regulation of $\beta$-catenin expression, and then improve myocardial function after myocardial infarction [7]. The expression of wnt3a, p-GSK-3 $\beta$ and $\beta$-catenin in ischemic-reperfused rat model were significantly higher than those in ischemia-reperfusion group, and the contents of wnt3a, p-GSK-3ßand p- B-catenin protein expression, and its effect in a concentration-dependent manner, 
suggesting that the total glucosides of paeony may inhibit the phosphorylation of GSK-3 $\beta$ by inhibiting the expression of wnt3a and play a preconditioning effect of ischemia-reperfusion, and Wnt3a, And the levels of $p-G S K-3 \beta$ and $\beta$-catenin protein were negatively correlated with the protection of myocardial ischemia-reperfusion injury.

Wnt signaling pathway is active during embryonic development, and has biphasic and antagonistic effects on myocardial formation and angiogenesis. In mature organism, this signal pathway is in a relatively static state, but can be re-expressed in some pathologic conditions. Myocardial infarction B-catenin also plays an important role in the remodeling of the mature myocardium and the healing of the myocardium after myocardial infarction [8]. The results of this experiment also suggest that the expression of $\beta$-catenin Total glycosides decreased myocardial infarct size and cardiomyocyte apoptosis induced by ischemia-reperfusion in a concentration-dependent manner, and correlated with inhibition of Wnt signaling and subsequent inhibition of $\beta$-catenin expression.

\section{Conclusion}

The total glucosides of paeony on ischemia-reperfusion injury can significantly improve the cardiac function and reduce the myocardial infarction and apoptosis after myocardial ischemia-reperfusion injury. The effect may be related to Wnt / $\beta$-catenin signal Pathways.

\section{Acknowledgements}

Fund Project: Henan Provincial Science and Technology Department Basic and Cutting-Edge Research Key Research Projects (No 162300410268)

\section{References}

[1] Boudina, S. and E.D. Abel, Diabetic cardiomyopathy, causes and effects[J]. Rev Endocr Metab Disord, 2010. 11(1): 31-39.

[2] Liu JW, Liu D, Cui KZ, et al. Recent advances in understanding the biochemical and molecular mechanism of diabetic cardiomyopathy[J]. Biochem Biophys Res Commun. 2012; 427(3):441-443.

[3] Huang Yiqian, Wang Xian, Kong Wei. Progress in the Pathogenesis of Diabetic Cardiomyopathy[J]. Advances in Physiological Sciences, 2010, 41 (1): 31-36.

[4] Vidal RL, Hetz C. Crosstalk between the UPR and autophagy pathway contributes to handling cellular stress in neurodegenerative disease[J]. Autophagy. 2012 Jun; 8(6):970-972.

[5] Kawamura M, Miyagawa S, Miki K, et al. Feasibility, safety, and therapeutic efficacy of human induced pluripotent stem cell-derived cardiomyocyte sheets in a porcine ischemic cardiomyopathy model[J]. Circulation, 2012, 126(11 Suppl 1): S29-37.

[6] Lian X, Hsiao C, Wilson G, et al. Robust cardiomyocyte differentiation from human pluripotent stem cells via temporal modulation of canonical Wnt signaling[J]. Proc Natl Acad Sci USA, 2012, 109(27): E1848-1857.

[7] Zhou X, Hu X, Xie J, et al. Exogenous high-mobility group box1 protein injection improves cardiac function after myocardial infarction: involvement of Wnt signaling activation[J]. $\mathrm{J}$ Biomed Biotechnol, 2012, 2012: 743879.

[8] ZelarayanL, GehrkeC, BergmannMW. Role of beta-eatenin in adult cardiac remodeling[J]. Cell Cycle, 2007, 6(17): 2120-2126. 